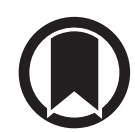

CrossMark

\title{
Radiographic pulmonary vessel volume, lung function and airways disease in the Framingham Heart Study
}

\author{
Andrew J. Synn $\mathbb{1}^{1,2}$, Wenyuan Li $\mathbb{B}^{3}$, Raúl San José Estépar $\mathbb{0}^{4}$, Chunyi Zhang ${ }^{1}$, \\ George R. Washko ${ }^{5,6}$, George T. 0'Connor ${ }^{6,7}$, Tetsuro Araki ${ }^{4}$, Hiroto Hatabu ${ }^{4,6}$, \\ Alexander A. Bankier ${ }^{8}$, Murray A. Mittleman ${ }^{3}$ and Mary B. Rice (i) 1
}

\begin{abstract}
Affiliations: 'Division of Pulmonary, Critical Care and Sleep Medicine, Beth Israel Deaconess Medical Center Harvard Medical School, Boston, MA, USA. ${ }^{2}$ Division of Pulmonary and Critical Care Medicine, Massachusetts General Hospital, Harvard Medical School, Boston, MA, USA. ${ }^{3}$ Dept of Epidemiology, Harvard T.H. Chan School of Public Health, Boston, MA, USA. ${ }^{4}$ Dept of Radiology, Brigham and Women's Hospital, Harvard Medical School, Boston, MA, USA. 'Division of Pulmonary and Critical Care Medicine, Brigham and Women's Hospital, Harvard Medical School, Boston, MA, USA. 'The NHLBI's Framingham Heart Study, Framingham, MA, USA. ${ }^{7}$ Pulmonary Center, Boston University School of Medicine, Boston, MA, USA. ${ }^{8}$ Dept of Radiology, Beth Israel Deaconess Medical Center, Harvard Medical School, Boston, MA, USA.
\end{abstract}

Correspondence: Andrew J. Synn, Division of Pulmonary, Critical Care and Sleep Medicine, Beth Israel Deaconess Medical Center, 330 Brookline Ave KSB-23, Boston, MA 02215-5491, USA. E-mail: asynnabidmc. harvard.edu

@ERSpublications

Lower total and peripheral pulmonary blood vessel volumes on CT are associated with worse lung health in a generally healthy population. CT imaging may be useful in detecting early changes in the pulmonary vessels, even before lung disease develops. http://bit.ly/2RyDLSp

Cite this article as: Synn AJ, Li W, San José Estépar R, et al. Radiographic pulmonary vessel volume, lung function and airways disease in the Framingham Heart Study. Eur Respir J 2019; 54: 1900408 [https://doi. org/10.1183/13993003.00408-2019].

ABSTRACT Radiographic abnormalities of the pulmonary vessels, such as vascular pruning, are common in advanced airways disease, but it is unknown if pulmonary vascular volumes are related to measures of lung health and airways disease in healthier populations.

In 2388 participants of the Framingham Heart Study computed tomography (CT) sub-study, we calculated total vessel volumes and the small vessel fraction using automated CT image analysis. We evaluated associations with measures of lung function, airflow obstruction on spirometry and emphysema on CT. We further tested if associations of vascular volumes with lung function were present among those with normal forced expiratory volume in $1 \mathrm{~s}$ and forced vital capacity.

In fully adjusted linear and logistic models, we found that lower total and small vessel volumes were consistently associated with worse measures of lung health, including lower spirometric volumes, lower diffusing capacity and/or higher odds of airflow obstruction. For example, each standard deviation lower small vessel fraction (indicating more severe pruning) was associated with a $37 \%$ greater odds of obstruction (OR 1.37, 95\% CI 1.11-1.71, p=0.004). A similar pattern was observed in the subset of participants with normal spirometry.

Lower total and small vessel pulmonary vascular volumes were associated with poorer measures of lung health and/or greater odds of airflow obstruction in this cohort of generally healthy adults without high burdens of smoking or airways disease. Our findings suggest that quantitative CT assessment may detect subtle pulmonary vasculopathy that occurs in the setting of subclinical and early pulmonary and airways pathology.

This article has supplementary material available from erj.ersjournals.com

Received: 27 Feb 2019 | Accepted after revision: 24 May 2019

Copyright (CERS 2019 


\section{Introduction}

Abnormalities of the pulmonary vessels are commonly seen across a number of pulmonary diseases, including chronic obstructive pulmonary disease (COPD), asthma, idiopathic pulmonary fibrosis, bronchiectasis and others [1-4]. For individuals with these conditions, the development of overt pulmonary vascular disease (known as Group 3 pulmonary hypertension in the World Health Organization classification scheme [5]) is a major clinical inflection point that predicts future morbidity and mortality [6-10]. It is likely that mild perturbations in the pulmonary vascular system, even below traditional thresholds of disease, are associated with poor outcomes [11, 12].

Assessment of the pulmonary vessels has historically required invasive angiography or tissue specimens [13]. As a result, the ability to study how pulmonary vascular abnormalities develop and progress in the setting of lung disease has been limited. Using computer-based image analysis of computed tomography (CT) scans, the total volume of the pulmonary blood vessels of varying sizes can now be assessed noninvasively [14]. By quantifying relative reductions in the volume of the smallest, most peripheral pulmonary vessels, this method can generate a CT surrogate of vascular pruning, which is known to occur angiographically in COPD [15-17]. In cohorts of individuals selected on the basis of airways disease, including COPD and asthma, radiographic pruning has been associated with the severity of lung disease, including poorer lung function, right ventricular dysfunction and higher pulmonary artery pressures $[1,2$, $14,18,19]$.

These results have generated new insights into the "vascular substance" of pulmonary disease, and raised the possibility that quantifying vascular abnormalities may play a role in phenotyping and stratifying populations with lung disease [20]. However, this work has primarily focused on cohorts specifically selected on the basis of clinically manifested airways disease and/or smoking. For the much larger number of individuals who have subclinical or early pulmonary pathology, it is unknown whether abnormalities of the pulmonary vessels are detectable by CT, and whether these vascular abnormalities are associated with poorer baseline lung function or more severe decline in lung function over time.

To address this knowledge gap, we investigated associations of absolute and relative pulmonary blood vessel volumes and measures of pulmonary function and airways disease in the Framingham Heart Study, a large, community-dwelling cohort without high burdens of smoking or lung disease. We hypothesised that more severe vascular pruning as shown by CT would be associated with poorer lung function and greater odds of airflow obstruction on spirometry or emphysema on CT.

\section{Materials and methods}

\section{Study population}

The study population consisted of participants of the Framingham Heart Study Offspring and Third Generation cohorts, described previously [21, 22]. From 2008 to 2011, 2764 participants underwent volumetric chest CT scans as part of the Multi-Detector Computed Tomography 2 sub-study, with the primary goal of assessing coronary artery calcification [23-25]. Of these, 2484 participants had satisfactory image data for measuring vascular morphology; those without demographic data or information on smoking history were excluded, leaving 2388 participants in the analysis. Most participants attended Framingham Offspring examination 8 (2005-2008) and Third Generation examination 2 (2008-2011), except nine participants (eight from Offspring) who attended Offspring examination 7 (1998-2001) or Third Generation examination 1 (2002-2005). Our sample included a sub-population of 842 Offspring participants who had repeated measures of spirometry data from examination 9 (2011-2014), which were compared to prior results to assess the trajectory of lung function. This study was conducted in accordance with the Declaration of Helsinki. The institutional review boards of the Beth Israel Deaconess Medical Center and Boston University Medical Center approved this study. All participants provided written informed consent.

\section{Radiographic pulmonary vascular assessment}

Inspiratory, non-contrast CT examinations covering the entire thorax were performed in the supine body position using a 64-detector-row scanner (Discovery, GE Healthcare, Waukesha, WI, USA), with uniform acquisition and reconstruction protocols. Acquisition parameters were $120 \mathrm{kVp}, 300-350 \mathrm{~mA}$ (based on body weight), $350 \mathrm{~ms}$ rotation time and $0.625 \mathrm{~mm}$ section thickness. Image analysis was performed in the Applied Chest Imaging Laboratory at Brigham and Women's Hospital utilising software based on the Chest Imaging Platform (www.chestimagingplatform.org) as previously described [1]. An automated algorithm generated three-dimensional reconstructions of the pulmonary vasculature from which the volumes of vessels of varying cross-sectional areas were calculated (figure 1), including the total volume of all intraparenchymal vessels (TBV) and of the small vessels (defined by cross-sectional area $<5 \mathrm{~mm}^{2}$ (BV5)). The measures generated by this algorithm reflect the volume of the entire vessel, including the vascular wall and lumen, and both arterial and venous vessels are included. The small vessel fraction 
FIGURE 1 a) Coronal chest computed tomography projection with overlaid volumetric reconstruction of the pulmonary vascular tree from a Framingham Heart Study participant. Vessels are colour-coded by size. b) Representative quantitative histogram illustrating the distribution of vascular volume as a function of vessel size.
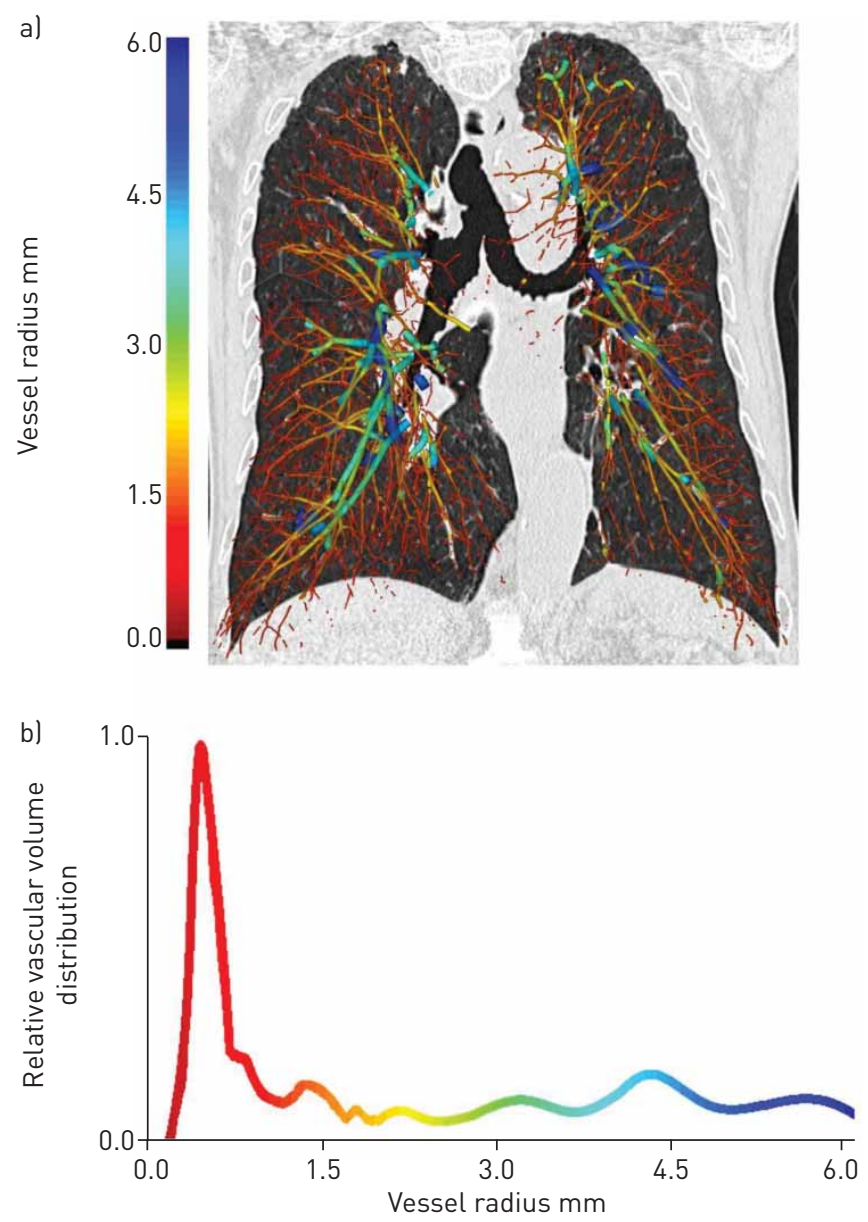

(BV5/TBV), representing the relative distribution of blood vessel volume in the smallest, most peripheral blood vessels detectable by CT, was calculated as a radiographic measure reflective of pulmonary vascular pruning $[1,2,26]$.

\section{Measures of pulmonary function and airways disease}

Lung function testing was performed at each Framingham Heart Study examination. In our sample of 2388 participants, 2255 (94.4\%) had spirometry results from a visit at the time of, or before, their respective CT scan. For Offspring examination 7, spirometric data were obtained using a Collins Survey II Water-Seal Spirometer (Warren Collins, Inc., Braintree, MA, USA) and acquisition and quality control software (S\&M Instruments, Doylestown, PA, USA), calibrated daily. For Offspring examinations 8 and 9, and Third Generation examinations 1 and 2, spirometric data were obtained using a Collins Comprehensive Pulmonary Laboratory system (nSpire Health, Inc., Longmont, CO, USA), calibrated daily. Spirometric manoeuvres were performed according to American Thoracic Society standards [27]. Measures of pulmonary function included forced expiratory volume in $1 \mathrm{~s}$ (FEV1), forced vital capacity (FVC), ratio of FEV1/FVC and diffusing capacity of the lung for carbon monoxide (DLCO). In a subset of the Offspring cohort that underwent follow-up lung function testing after CT scan, we calculated the annualised change in FEV1, FVC and DLCO to assess the trajectory of lung function.

Measures of airways disease included airflow obstruction on spirometry and visual appearance of emphysema on CT. Obstruction was defined as moderate-to-severe airflow limitation based on an FEV1/ FVC ratio $<0.70$ with an $\mathrm{FEV}_{1} \%$ predicted $<80 \%$ [28]. Emphysema was determined based on visual evaluation by three board-certified radiologists using a modified sequential reading method as previously described [23, 29, 30], and results were available for 2291 participants (95.9\%). This assessment was performed on the same CT scan that was used to generate the pulmonary vascular volumes.

\section{Statistical methods}

Multivariable linear regression models were used to examine associations of CT vascular measures (TBV, BV5 and BV5/TBV) with continuous pulmonary outcomes (FEV1, FVC, DLCO and annualised change in 
these measures), and multivariable logistic regression models were used for binary outcomes (airflow obstruction on spirometry and visual emphysema on CT). We evaluated departures from linearity for the association of CT vascular measures and lung function by plotting penalised splines with generalised additive models with a Gaussian distribution, and used the likelihood ratio test to compare the nested models. All models included covariates that were selected a priori based on known or suspected associations with airways disease, thoracic size or abnormalities of pulmonary vessels. These included age at time of CT, sex, height, weight, smoking status (current, former or never), total cumulative pack-years of cigarette exposure, personal educational attainment [30], occupation (labourer, sales/clerical, professional/technical, executive/supervisory or other) [31], census tract median neighbourhood income (using home address at visit date and year 2005-2009 census data) [31] and cohort identifier. We did not adjust for race/ethnicity because nearly all participants are of European ancestry [32].

In secondary analyses, we added interactions terms to our primary models to test for evidence of differential associations of radiographic pulmonary blood vessel volumes with measures of pulmonary function and airways disease based on ever-smoking status. For continuous measures of lung function, we performed a sensitivity analysis assessing the association of radiographic pulmonary vascular volumes and FEV1, FVC and DLCO in the 1984 participants (83.1\%) who had normal spirometric volumes (i.e. FEV1 \% pred and FVC \% pred both $\geqslant 80 \%$ ). Regression coefficients and odds ratios are reported with $95 \%$ confidence intervals and expressed per standard deviation difference in the vascular parameter. For interaction terms, a two-sided p-value of $<0.10$ for the Wald test was considered a statistically significant effect modification. Statistical analyses were performed using SAS 9.4 (SAS Institute, Inc., Cary, NC, USA) and penalised spline plots were produced using $\mathrm{R}$ version 3.5.1 ( $\mathrm{R}$ Foundation for Statistical Computing, Vienna, Austria).

\section{Results}

\section{Study participant characteristics}

Details of the study cohort are provided in table 1 . The mean age of the cohort was $59.0 \pm 11.7$ years and $51.1 \%$ were female. Nearly half the participants (48.9\%) were never-smokers, while $43.7 \%$ were former smokers and $7.4 \%$ were current smokers. Mean lung function in the cohort was normal, with mean $\%$ pred FEV 1, FVC and DLCO of $97.9 \pm 15.2 \%, 101.8 \pm 13.3 \%$ and $96.6 \pm 15.7 \%$, respectively. The mean FEV $1 / F V_{C}$ was $74.2 \pm 7.3 \%$. In the Offspring subset that had follow-up spirometry, over an average interval of $5.7 \pm 0.4$ years, FEV1, FVC and DLCO declined by a mean of $33.0 \pm 36.4 \mathrm{~mL} \cdot \mathrm{year}^{-1}, 45.4 \pm 46.6 \mathrm{~mL} \cdot \mathrm{year}^{-1}$ and $0.36 \pm 0.52 \mathrm{~mL} \cdot \mathrm{min}^{-1} \cdot \mathrm{mmHg}^{-1} \cdot \mathrm{year}^{-1}$, respectively. For measures of airways disease, 167 participants $(7.4 \%)$ met criteria for moderate-to-severe obstruction on spirometry, while 297 (13.0\%) had CT evidence of emphysema.

Mean TBV and BV5 were $143.1 \pm 30.8 \mathrm{~mL}$ and $55.9 \pm 11.5 \mathrm{~mL}$, respectively, and the small vessel fraction (BV5/TBV) was $39.3 \pm 4.1 \%$. Participants with airflow obstruction had similar TBV, but lower BV5 and BV5/TBV compared to those without obstruction (supplementary table S1).

\section{Associations with measures of pulmonary function and airways disease}

Associations of pulmonary blood vessel volumes with measures of pulmonary function and airways disease are reported in tables 2 and 3. Lower blood vessel volume was consistently associated with worse pulmonary function in fully adjusted models. For example, per SD lower TBV, FEV1 was $145.1 \mathrm{~mL}$ lower (95\% CI 117.6-172.6, p<0.0001), FVC was $248.8 \mathrm{~mL}$ lower (95\% CI 217.6-280.0, p <0.0001) (figure 2) and DLCO was $1.40 \mathrm{~mL} \cdot \mathrm{min}^{-1} \cdot \mathrm{mmHg}^{-1}$ lower (95\% CI $1.16-1.63, \mathrm{p}<0.0001$ ) (figure 3 ). A similar pattern was observed for the small vessel fraction: more severe pruning (i.e. lower BV5/TBV) was associated with lower FEV1 and FVC, although not with DLCO (figures 2 and 3). We did not find any associations of radiographic pulmonary vascular volumes with annualised change in FEV1, FVC or DLCO (table 2). The penalised splines for the associations of TBV and BV5 with continuous measures of lung function (FEV1, FVC and DLCO) showed linear relationships (supplementary figures S1 and S2). In addition, the spline of the association of BV5/TBV with FEV1 and FVC was roughly linear (supplementary figure S3).

For measures of airways disease on spirometry and CT, we found that more severe CT pruning was associated with higher odds of obstruction in fully adjusted models (table 3); each sD lower BV5/TBV was associated with $37 \%$ higher odds of obstruction (OR 1.37, 95\% CI 1.11-1.71, p=0.004). There was no association of CT pulmonary vascular volumes and emphysema on CT (figure 4).

\section{Secondary analyses}

In secondary analyses, we found no evidence that the associations of radiographic pulmonary vascular volumes with spirometry or trajectory of lung function differed by ever-smoking status (all $\mathrm{p}_{\text {interaction }}>0.10$ ). For associations of absolute CT vascular volumes (TBV and BV5) and the presence of 
TABLE 1 Characteristics of study participants

\section{Characteristic}

\begin{tabular}{lc}
\hline Subjects $\mathbf{n}$ & 2388 \\
Age years & $59.0 \pm 11.7$ \\
Female & $1219(51.1)$ \\
Body-mass index kg.m ${ }^{-2}$ & $28.4 \pm 5.4$ \\
Occupation category & $191(8.0)$ \\
$\quad$ Labourer & $609(25.5)$ \\
Sales/clerical & $989(41.4)$ \\
Professional/Executive/Supervisory/Technical & $599(25.1)$ \\
Other & \\
Educational attainment & $504(21.1)$ \\
High school or less & $758(31.7)$ \\
Some college & $1126(47.2)$ \\
College/grad school & $83065(36801)$ \\
Census tract income US\$ median (IQR) & $1093(45.8)$ \\
Offspring cohort & \\
Smoking status & $1168(48.9)$ \\
$\quad$ Never & $1043(43.7)$ \\
Former & $177(7.4)$ \\
Current & \\
Pack-years of smoking & $9.8 \pm 15.7$ \\
Entire sample & $16.9 \pm 16.7$ \\
Former smokers & $32.7 \pm 15.5$ \\
Current smokers & $97.9 \pm 15.2$ \\
FEV1 \% pred & $101.8 \pm 13.3$ \\
FVC \% pred & $96.6 \pm 15.7$ \\
DLco \% pred & $167(7.4)$ \\
Airflow obstruction on spirometry & $297(13.0)$ \\
Emphysema on CT & \\
CT pulmonary vascular measures & $143.1 \pm 30.8$ \\
TBV mL & $55.9 \pm 11.5$ \\
BV5 mL & $39.3 \pm 4.1$ \\
BV5/TBV \% & \\
\end{tabular}

Data are presented as mean \pm SD or $\mathrm{n}(\%)$, unless otherwise stated. IQR: interquartile range; FEV1: forced expiratory volume in $1 \mathrm{~s}$ : FVC: forced vital capacity; DLCO: diffusing capacity of the lung for carbon monoxide; CT: computed tomography; TBV: total blood vessel volume; BV5: blood vessels with cross-sectional area $<5 \mathrm{~mm}^{2}$.

TABLE 2 Associations of computed tomographic pulmonary vascular volumes and measures of pulmonary function

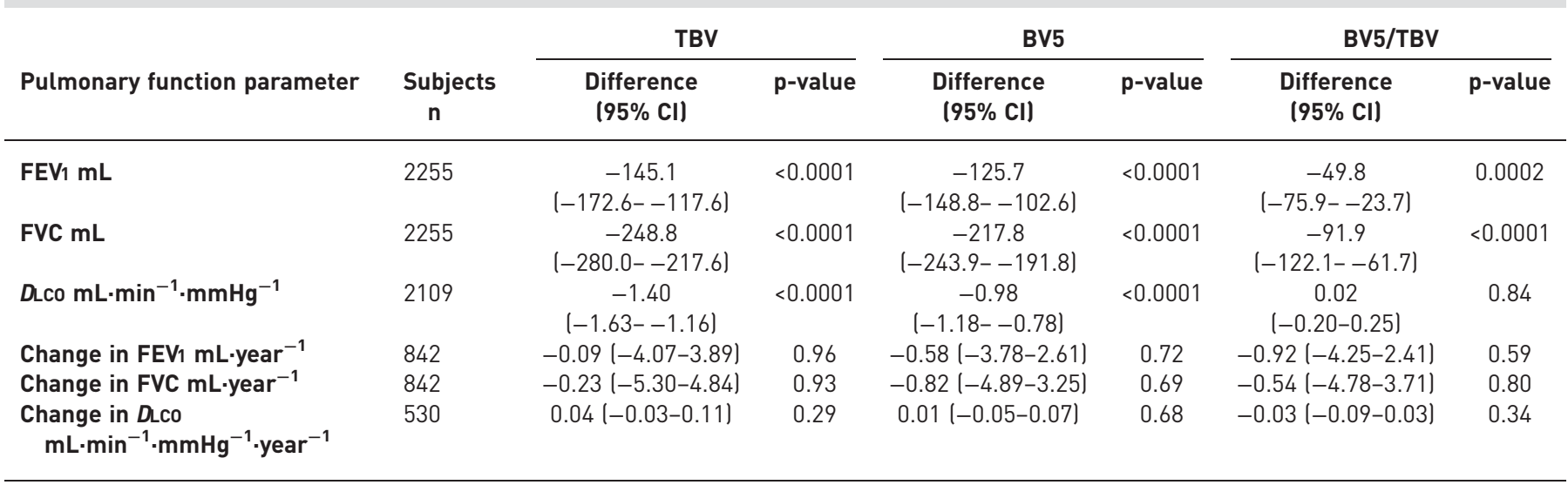

Results of linear regression models with adjustment for age, sex, height, weight, smoking status, total pack-years of cigarette exposure, personal educational attainment, occupation category, census tract median neighbourhood income and Framingham Heart Study cohort (Offspring versus Third Generation). Data are expressed per SD lower blood vascular parameter. TBV: total blood vessel volume; BV5: blood vessels with cross-sectional area $<5 \mathrm{~mm}^{2}$; FEV1: forced expiratory volume in $1 \mathrm{~s}$ : FVC: forced vital capacity; DLco: diffusing capacity of the lung for carbon monoxide. 
TABLE 3 Associations of radiographic computed tomographic pulmonary vascular volumes and odds of airways disease

\begin{tabular}{|c|c|c|c|c|c|c|c|}
\hline \multirow[t]{2}{*}{ Airways disease parameter } & \multirow[t]{2}{*}{ Subjects $n$} & \multicolumn{2}{|l|}{ TBV } & \multicolumn{2}{|l|}{ BV5 } & \multicolumn{2}{|c|}{ BV5/TBV } \\
\hline & & OR $(95 \% \mathrm{CI})$ & p-value & OR $(95 \% \mathrm{CI})$ & p-value & OR $(95 \% \mathrm{CI})$ & p-value \\
\hline Presence of obstruction on spirometry & 2255 & $1.03(0.80-1.32)$ & 0.82 & $1.21(0.98-1.49)$ & 0.08 & $1.37(1.11-1.71)$ & 0.004 \\
\hline
\end{tabular}

Results of logistic regression models with adjustment for age, sex, height, weight, smoking status, total pack-years of cigarette exposure, personal educational attainment, occupation category, census tract median neighbourhood income and Framingham Heart Study cohort (Offspring versus Third Generation). Data are expressed per SD lower blood vascular parameter. TBV: total blood vessel volume; BV5: blood vessels with cross-sectional area $<5 \mathrm{~mm}^{2} ; \mathrm{CT}$ : computed tomography.

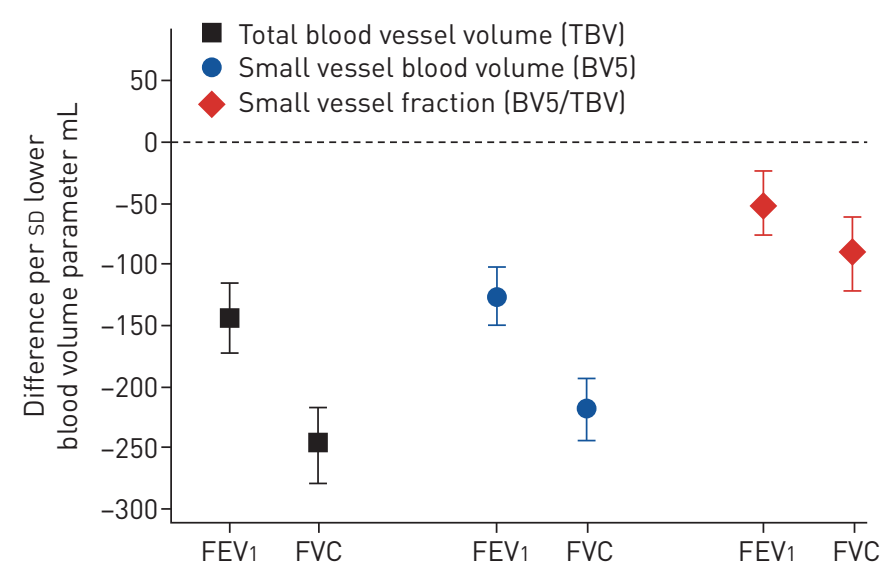

FIGURE 2 Associations of computed tomographic pulmonary vascular volumes with spirometry lexpressed per SD lower vascular volume parameter). Results of linear regression models (expressed per SD lower vascular volume parameter) with adjustment for age, sex, height, weight, smoking status, total pack-years of cigarette exposure, personal educational attainment, occupation category, census tract median neighbourhood income and Framingham Heart Study cohort (Offspring versus Third Generation). BV5: blood vessels with cross-sectional area $<5 \mathrm{~mm}^{2}$; FEV1: forced expiratory volume in $1 \mathrm{~s}$; FVC: forced vital capacity.

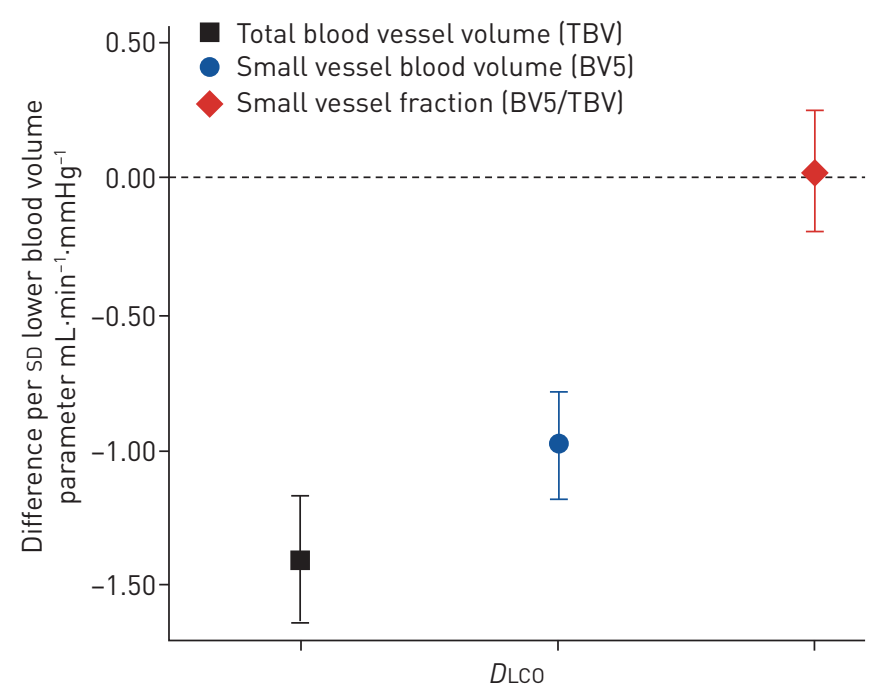

FIGURE 3 Associations of computed tomographic pulmonary vascular volumes with diffusing capacity of the lung for carbon monoxide ( $D$ LCO) (expressed per SD lower vascular parameter). Results of linear regression models (expressed per SD lower vascular volume parameter) with adjustment for age, sex, height, weight, smoking status, total pack-years of cigarette exposure, personal educational attainment, occupation category, census tract median neighbourhood income and Framingham Heart Study cohort lOffspring versus Third Generation). BV5: blood vessels with cross-sectional area $<5 \mathrm{~mm}^{2}$. 


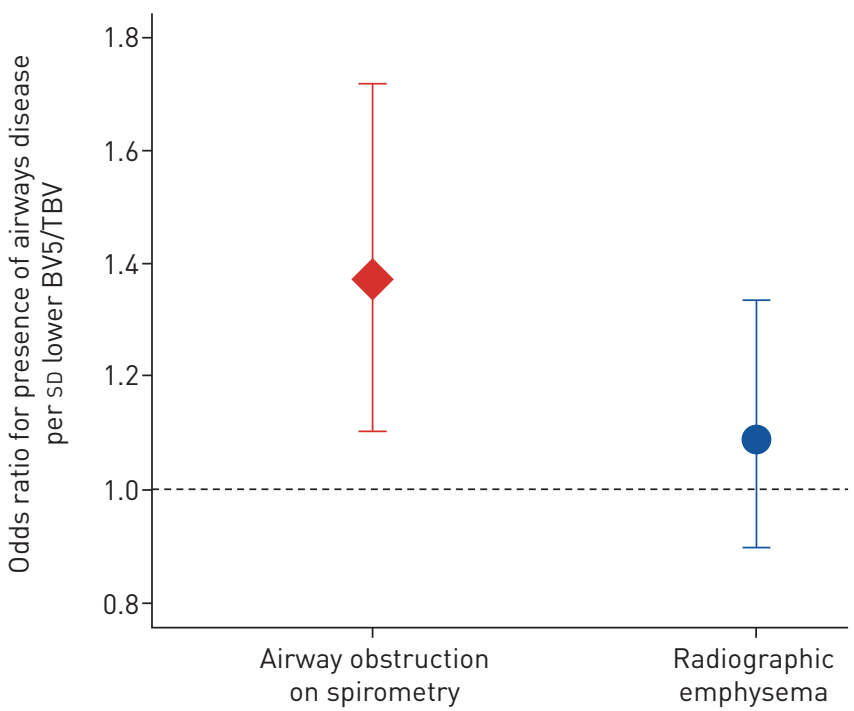

FIGURE 4 Associations of computed tomographic pulmonary vascular pruning with airways disease. Results of logistic regression models (expressed per SD lower BV5/TBV) with adjustment for age, sex, height, weight, smoking status, total pack-years of cigarette exposure, personal educational attainment, occupation category, census tract median neighbourhood income and Framingham Heart Study cohort lOffspring versus Third Generation). BV5: blood vessels with cross-sectional area $<5 \mathrm{~mm}^{2}$; TBV: total blood vessel volume.

airways disease defined by spirometry or CT, we found inconsistent evidence of differential relationships based on smoking status. In the case of emphysema on CT, in ever-smokers there was an association with higher odds of emphysema per SD higher TBV (OR 1.26, 95\% CI 1.01-1.57), and this relationship was not seen in never-smokers ( $p_{\text {interaction }}=0.03$ ). There was no evidence that the associations of CT pruning (BV5/ TBV) with pulmonary function and airways disease differed by smoking status (all $p_{\text {interaction }}>0.10$ ).

In a sensitivity analysis restricted to participants with normal FEV1 and FVC ( $\geqslant 80 \%$ pred), the pattern of associations of CT pulmonary blood vessel volumes and lung function was generally consistent. Associations of TBV and BV5 with FEV1, FVC and DLCO remained significant (all $\mathrm{p}<0.0001$ ). For small vessel fraction, BV5/TBV was associated with FVC $(\mathrm{p}<0.0001)$, but the association with $\mathrm{FEV} 1$ was attenuated $(\mathrm{p}=0.099)$.

\section{Discussion}

In this large cohort of community-dwelling and generally healthy adults, we found that more severe vascular pruning and lower pulmonary blood vessel volumes derived from automated CT image analysis were consistently associated with worse measures of lung health, including lower spirometric volumes and diffusing capacity, and greater odds of airflow obstruction on spirometry. These associations persisted when restricting the analysis to participants with normal spirometry, and did not substantially differ based on the presence of ever-smoking exposure. This suggests that, even in populations in which only a minority of individuals have abnormal lung function or airways disease, differences in pulmonary vascular volumes detectable by CT are associated with indicators of lung health.

These results are consistent with previous work in cohorts of individuals with substantial airways disease, including COPDGene and the Severe Asthma Research Program, which also utilised a similar quantitative vascular image analysis technique as our study $[1,2]$. These studies have primarily focused on the relative volume of the smallest pulmonary vessels (the BV5/TBV fraction) as an indicator of the angiographic pruning that is known to occur in COPD. In these studies, a lower BV5/TBV (indicating more severe CT pruning) was consistently associated with more severe airways disease, including worse spirometry, higher Global Initiative for Chronic Obstructive Lung Disease (GOLD) stage, higher peripheral and sputum eosinophil percentage, higher odds of future asthma exacerbation during follow-up, presence of right ventricular dysfunction on cardiac magnetic resonance imaging (which may be a haemodynamic consequence of pruning and loss of the pulmonary vascular bed) and greater mortality during follow-up $[1,2,19,33]$. In a univariate analysis of participants of COPDGene, more severe CT pruning correlated with a higher percentage of low-attenuation area on CT, a quantitative densitometric measure of emphysema. In contrast, we found that CT pruning was not associated with the presence of visually evident emphysema on CT, which may be explained in part by the low burden of emphysema in the generally healthy Framingham cohort compared to the COPDGene cohort. The use of quantitative 
densitometric measures, which may potentially provide complementary information regarding emphysematous changes, may have helped to clarify this discrepancy; however, the parameters used for image acquisition and reconstruction in Framingham resulted in substantial artefact in the estimated percentage of low-attenuation area of the lung [30], which limits the use of these measures in our analyses.

A related, but distinct, method of quantifying pulmonary vascular pruning on CT has also been reported in the literature. This method calculates the relative cross-sectional area, rather than volume, of the small pulmonary vessels from three individual CT slices (one each from the upper, middle and lower sections of the lung). Using this area-based metric of vascular pruning, studies of individuals with COPD found that more severe pruning was associated with lower FEV1 and DLCO, and a greater extent of emphysema (as defined by the percentage of attenuation values lower than -950 Hounsfield units on each CT slice) [14, 18]. In a small group of 30 non-smoking people without airflow obstruction on spirometry, area-based pulmonary vascular pruning on CT was not associated with lower FEV1 [34]. However, this was a univariate analysis in a small number of individuals, and does not necessarily contradict our finding that healthy non-smokers with lower BV5/TBV had worse lung function, on average.

Our study also adds to the more general body of literature utilising CT imaging techniques to study the earliest pathologic changes in airways disease. Recently, Koo et al. [35] used an innovative combination of conventional CT, micro-CT and histology to show that individuals with mild and moderate COPD had decreased numbers of small airways compared to a control group of smokers with normal lung function. Furthermore, when examining only tissue samples without emphysematous destruction (as defined by normal alveolar surface area on micro-CT), those with mild and moderate COPD still had fewer small airways compared to controls.

The remodelling and loss of airways in smokers with early COPD thus parallels the pulmonary vascular changes identified in smokers. SANTOS et al. [36] demonstrated that structural abnormalities of the small pulmonary arteries were evident in mild COPD, and even smokers without airflow obstruction, who would have been included in the control group in many COPD studies, had similar vascular morphometric characteristics. In guinea pig models, tobacco exposure induced pulmonary vascular alterations, including early structural remodelling, which were seen before emphysematous changes occurred [37, 38]. Our results contribute to this existing literature by demonstrating that lower pulmonary vascular volumes, particularly in the smallest detectable vessels, are associated with poorer spirometric lung function (lower FEV1 and FVC), even when examining only those individuals with normal lung function. In addition, we found that these associations did not differ in smokers compared to non-smokers, which suggests that vascular abnormalities are also clinically relevant in the substantial minority of individuals with COPD who have not been exposed to tobacco [39], a population in which early pathologic changes are not well described.

Taken in context, the results of our study suggest that lower pulmonary blood vessel volumes may be an independent indicator of worse respiratory health, not only in individuals with substantial lung disease that might be expected to be at high risk for vascular pathology, but also in populations with normal average lung function and without high burdens of tobacco exposure. Our findings indicate that pulmonary vasculopathy (including vessel narrowing and obliteration) likely occurs alongside the loss of small airways in early airways disease, including in those individuals in whom the traditional disease criteria have not yet been fulfilled. Further work is necessary to determine the temporality of this association, which cannot be determined from our results. We believe that these observations underline the potential importance of vascular volumes on CT as an imaging biomarker.

However, our findings should also be considered alongside our prior study [26], in which we found that tobacco exposure was associated with higher total and small vessel volumes on CT, both in those with and without airflow obstruction. This result was unexpected given that histological results from humans and animal models demonstrated that tobacco exposure was associated with narrowing and obliteration of the small vessels. The exact explanation for this finding remains unclear: this association was robust to adjustment for a number of possible structural and functional factors (including airflow obstruction and cardiovascular disease), was consistent across multiple metrics of tobacco exposure, and was similar to a prior finding that was previously described in the Multi-Ethnic Study of Atherosclerosis (MESA) [26, 40].

In contrast, in the present analysis we found an association of more severe CT-based vascular pruning with poorer lung health using models that were adjusted for both smoking status and total pack-years of cigarette exposure. In addition, in secondary analyses to test for effect modification by smoking status, we also found no evidence that our primary results were different in smokers compared to non-smokers. These results indicate that CT measures of pruning are independent indicators of structural vascular changes associated with airways disease, including narrowing and loss of the small pulmonary vessels. However, given that tobacco exposure did not appear to manifest as straightforward pruning on CT in this 
same generally healthy population, we would caution that any analyses of structural vascular measures on CT must consider adjustment for cigarette smoke exposure. Further work is necessary to elucidate the factors underlying this potential discrepancy, and to ensure the consistent interpretation of similar vascular imaging measures in future analyses.

To our knowledge, our study is among the first to investigate associations of pulmonary vascular volumes on CT with pulmonary function and airways disease in a generally healthy population not selected on the basis of smoking or lung disease, and is also one of the largest to examine quantitative image analysis of the pulmonary vasculature. Strengths of our study include the large cohort size, with adjustment for a more detailed set of potential confounders than other reports in the literature. Additionally, our analyses included both absolute and relative pulmonary blood vessel volumes to assess differences in the distribution of the vasculature, and generated these volumes from all the pulmonary vessels visible on modern CT imaging, which may provide a more comprehensive vascular profile compared to methods that assess individual sections in isolation. One particular strength is that all CT was performed on the same model scanner, at one institution, and using a uniform protocol, thereby reducing the bias introduced by differences in image acquisition and manufacturer-related reconstruction algorithms that is a significant concern for radiographic biomarkers [41].

Our study has several limitations. The cross-sectional study design of our main analysis limits conclusions regarding the causality of the associations. Although our models adjusted for many potential confounders, residual confounding cannot be excluded. While the inspiratory, whole-lung CT scans enable interpretation of lung and pulmonary vascular images, a potential limitation of the imaging protocol is that the CT scans were gated to optimise assessment of coronary artery calcification. Due to the original recruitment strategy targeting residents of the town of Framingham in Massachusetts, USA, our sample is entirely of European ancestry, and our findings may not be generalisable to the general population.

Fundamental questions about the relationship between the pulmonary vascular system and airways disease remain unanswered. These include whether vascular abnormalities are passive changes secondary to an underlying non-vascular process, or whether they instead represent a key driver of COPD/emphysema, as has been suggested by histological studies of a small series of human smokers and animal models [36-38]. Additionally, given that loss of pulmonary arterioles and small airways have each been shown to occur prior to emphysematous tissue loss $[35,38]$, it is possible that early COPD is characterised by vasculopathy that is more closely linked to abnormalities in the small airways rather than the airspaces. Our finding that differences in radiographic blood vessel volumes are detectable on CT and are associated with lung health even in the absence of pulmonary disease is a novel contribution to the existing literature. These results support the expanded use of this technique to study and potentially identify the early pulmonary vascular changes that contribute to poorer long-term outcomes, which may represent a target for disease-modifying interventions. In addition, given that this method of vascular quantification has within-subject reproducibility [1] and can detect responses to treatment [42], it may be possible to use this technology to study how very early pulmonary vascular changes develop over time. Future directions for research in generally healthy cohorts such as Framingham may address associations of pulmonary vascular volumes with right-sided cardiac function, the relationship between central pulmonary vascular measures (such as the pulmonary artery-to-aorta ratio) and peripheral vascular ratios (including BV5/TBV), associations with quantitative measures of emphysema, and the impact of longitudinal pulmonary vascular changes on cardiopulmonary outcomes.

\section{Conclusions}

In this study of community-dwelling, generally healthy adults without high burdens of smoking or airways disease, lower total and peripheral vessel pulmonary blood vessel volumes were associated with poorer measures of lung health and/or greater odds of airflow obstruction. This suggests that quantitative CT assessment can be used to detect subtle pulmonary vasculopathy that occurs in the setting of subclinical and early pulmonary pathology.

Conflict of interest: A.J. Synn reports grants from NHLBI during the conduct of the study. W. Li has nothing to disclose. R. San José Estépar reports grants from NHLBI, during the conduct of the study; personal fees from Toshiba and Boehringer Ingelheim, outside the submitted work; and is also a founder and co-owner of Quantitative Imaging Solutions, a company that provides image-based consulting and develops software to enable data sharing. C. Zhang has nothing to disclose. G.R. Washko reports grants from NIH and BTG Interventional Medicine, grants from, provided consultancy to and participated on advisory boards for Boehringer Ingelheim, provided consultancy to and participated on data monitoring committees for PulmonX, grants from and provided consultancy to Janssen Pharmaceuticals, and provided consultancy to GlaxoSmithKline, outside the submitted work; G.R. Washko is a founder and co-owner of Quantitative Imaging Solutions, which provides image-based consulting and develops software to enable data sharing; and G.R. Washko's spouse works for Biogen, which is focused on developing therapies for fibrotic lung disease. G.T. O'Connor discloses grant support from $\mathrm{NIH}$, along with consulting fees from AstraZeneca and grant support from 
Jannsen Pharmaceuticals. T. Araki has nothing to disclose. H. Hatabu reports grants from Canon Medical Inc. and Konica-Minolta Inc., and personal fees for consultancy from Mitsubishi Chemical Inc., outside the submitted work. A.A. Bankier reports personal fees for consultancy from Spiration, Daiichi and Crico, and personal fees for authorship from Elsevier, outside the submitted work. M.A. Mittleman reports grants from NIH and US EPA, during the conduct of the study; and grants from NIH, US EPA, PCORI, US Dept of Justice and Kellogg Foundation, outside the submitted work. M.B. Rice reports grants from NIEHS during the conduct of the study.

Support statement: This study was supported by the American Lung Association, the American Thoracic Society, the National Institutes of Health/National Heart, Lung, and Blood Institute (1R01 HL116931, F32 HL143819, HHSN268201500001I, N01-HC-25195, R01 HL116473, R01 HL122464) and the National Institutes of Health/National Institute of Environmental Health Sciences (K23 ES026204). Funding information for this article has been deposited with the Crossref Funder Registry.

\section{References}

1 Estépar RSJ, Kinney GL, Black-Shinn JL, et al. Computed tomographic measures of pulmonary vascular morphology in smokers and their clinical implications. Am J Respir Crit Care Med 2013; 188: 231-239.

2 Ash SY, Rahaghi FN, Come CE, et al. Pruning of the pulmonary vasculature in asthma. The Severe Asthma Research Program (SARP) cohort. Am J Respir Crit Care Med 2018; 198: 39-50.

3 Jacob J, Bartholmai BJ, Rajagopalan S, et al. Mortality prediction in idiopathic pulmonary fibrosis: evaluation of computer-based CT analysis with conventional severity measures. Eur Respir J 2017; 49: 1601011.

4 Diaz AA, Maselli DJ, Rahaghi F, et al. Pulmonary vascular pruning in smokers with bronchiectasis. ERJ Open Res 2018; 4: 00044-2018.

5 Simonneau G, Gatzoulis MA, Adatia I, et al. Updated clinical classification of pulmonary hypertension. J Am Coll Cardiol 2013; 62: D34-D41.

6 Ghigna MR, Mooi WJ, Grünberg K. Pulmonary hypertensive vasculopathy in parenchymal lung diseases and/or hypoxia. Eur Respir Rev 2017; 26: 170003.

7 Klinger JR. Group III pulmonary hypertension: pulmonary hypertension associated with lung disease: epidemiology, pathophysiology, and treatments. Cardiol Clin 2016; 34: 413-433.

8 Thiwanka Wijeratne D, Lajkosz K, Brogly SB, et al. Increasing incidence and prevalence of World Health Organization groups 1 to 4 pulmonary hypertension: a population-based cohort study in Ontario, Canada. Circ Cardiovasc Qual Outcomes 2018; 11: e003973.

9 Oswald-Mammosser M, Weitzenblum E, Quoix E, et al. Prognostic factors in COPD patients receiving long-term oxygen therapy: importance of pulmonary artery pressure. Chest 1995; 107: 1193-1198.

10 Lettieri CJ, Nathan SD, Barnett SD, et al. Prevalence and outcomes of pulmonary arterial hypertension in advanced idiopathic pulmonary fibrosis. Chest 2006; 129: 746-752.

11 Maron BA, Hess E, Maddox TM, et al. Association of borderline pulmonary hypertension with mortality and hospitalization in a large patient cohort: insights from the Veterans Affairs clinical assessment, reporting, and tracking program. Circulation 2016; 133: 1240-1248.

12 Douschan P, Kovacs G, Avian A, et al. Mild elevation of pulmonary arterial pressure as a predictor of mortality. Am J Respir Crit Care Med 2018; 197: 509-516.

13 Voelkel NF, Gomez-Arroyo J, Mizuno S. COPD/emphysema: the vascular story. Pulm Circ 2011; 1: 320-326.

14 Matsuoka S, Washko GR, Yamashiro T, et al. Pulmonary hypertension and computed tomography measurement of small pulmonary vessels in severe emphysema. Am J Respir Crit Care Med 2010; 181: 218-225.

15 Scarrow GD. The pulmonary angiogram in chronic bronchitis and emphysema. Clin Radiol 1966; 17: 54-67.

16 Cordasco EM, Beerel FR, Vance JW, et al. Newer aspects of the pulmonary vasculature in chronic lung disease: a comparative study. Angiology 1968; 19: 399-407.

17 Jacobson G, Turner AF, Balchum OJ, et al. Vascular changes in pulmonary emphysema. The radiologic evaluation by selective and peripheral pulmonary wedge angiography. Am J Roentgenol Radium Ther Nucl Med 1967; 100: 374-396.

18 Matsuoka S, Washko GR, Dransfield MT, et al. Quantitative CT measurement of cross-sectional area of small pulmonary vessel in COPD. Correlations with emphysema and airflow limitation. Acad Radiol 2010; 17: 93-99.

19 Wells JM, Iyer AS, Rahaghi FN, et al. Pulmonary artery enlargement is associated with right ventricular dysfunction and loss of blood volume in small pulmonary vessels in chronic obstructive pulmonary disease. Circ Cardiovasc Imaging 2015; 8: e002546.

20 Sverzellati N, Silva M. The matter of the lung: quantification of vascular substance in asthma. Am J Respir Crit Care Med 2018; 198: 1-2.

21 Kannel WB, Feinleib M, Mcnamara PM, et al. An investigation of coronary heart disease in families: the Framingham Offspring study. Am J Epidemiol 1979; 110: 281-290.

22 Splansky GL, Corey D, Yang Q, et al. The Third Generation cohort of the National Heart, Lung, and Blood Institute's Framingham Heart Study: design, recruitment, and initial examination. Am J Epidemiol 2007; 165: $1328-1335$.

23 Araki T, Nishino M, Zazueta OE, et al. Paraseptal emphysema: prevalence and distribution on CT and association with interstitial lung abnormalities. Eur J Radiol 2015; 84: 1413-1418.

24 Kliment CR, Araki T, Doyle TJ, et al. A comparison of visual and quantitative methods to identify interstitial lung abnormalities. BMC Pulm Med 2015; 15: 134

25 Dorans KS, Wilker EH, $\mathrm{Li} \mathrm{W}$, et al. Residential proximity to major roads, exposure to fine particulate matter, and coronary artery calcium. Arterioscler Thromb Vasc Biol 2016; 36: 1679-1685.

26 Synn AJ, Zhang C, Washko GR, et al. Cigarette smoke exposure and radiographic pulmonary vascular morphology in the Framingham Heart Study. Ann Am Thorac Soc 2019; 16: 698-706.

27 Standardization of spirometry: 1994 Update. American Thoracic Society. Am J Respir Crit Care Med 1995; 152: $1107-1136$.

28 Vogelmeier CF, Criner GJ, Martinez FJ, et al. Global strategy for the diagnosis, management, and prevention of chronic obstructive lung disease 2017 report. GOLD executive summary. Am J Respir Crit Care Med 2017; 195: 557-582. 
29 Washko GR, Lynch DA, Matsuoka S, et al. Identification of early interstitial lung disease in smokers from the COPDGene study. Acad Radiol 2010; 17: 48-53.

30 Rice MB, Li W, Dorans KS, et al. Exposure to traffic emissions and fine particulate matter and computed tomography measures of the lung and airways. Epidemiology 2018; 29: 1-4.

$31 \mathrm{Li}$ W, Dorans KS, Wilker EH, et al. Short-term exposure to ambient air pollution and biomarkers of systemic inflammation: the Framingham Heart Study. Arterioscler Thromb Vasc Biol 2017; 37: 1793-1800.

32 Tsao CW, Vasan RS. Cohort profile: the Framingham Heart Study (FHS): overview of milestones in cardiovascular epidemiology. Int J Epidemiol 2015; 44: 1800-1813.

33 Washko GR, Nardelli P, Ash SY, et al. Arterial vascular pruning, right ventricular size and clinical outcomes in COPD. Am J Respir Crit Care Med 2019; 200: 454-461.

34 Uejima I, Matsuoka S, Yamashiro T, et al. Quantitative computed tomographic measurement of a cross-sectional area of a small pulmonary vessel in nonsmokers without airflow limitation. Jpn J Radiol 2011; 29: 251-255.

35 Koo HK, Vasilescu DM, Booth S, et al. Small airways disease in mild and moderate chronic obstructive pulmonary disease: a cross-sectional study. Lancet Respir Med 2018; 6: 591-602.

36 Santos S, Peinado VI, Ramírez J, et al. Characterization of pulmonary vascular remodelling in smokers and patients with mild COPD. Eur Respir J 2002; 19: 632-638.

37 Wright JL, Churg A. Effect of long-term cigarette smoke exposure on pulmonary vascular structure and function in the Guinea pig. Exp Lung Res 1991; 17: 997-1009.

38 Ferrer E, Peinado VI, Díez M, et al. Effects of cigarette smoke on endothelial function of pulmonary arteries in the guinea pig. Respir Res 2009; 10: 76.

39 Lamprecht B, McBurnie MA, Vollmer WM, et al. COPD in never smokers: results from the population-based burden of obstructive lung disease study. Chest 2011; 139: 752-763.

40 Aaron CP, Hoffman EA, Lima JAC, et al. Pulmonary vascular volume, impaired left ventricular filling and dyspnea: the MESA Lung Study. PLoS One 2017; 12: e0176180.

41 Bodduluri S, Reinhardt JM, Hoffman EA, et al. Recent advances in computed tomography imaging in chronic obstructive pulmonary disease. Ann Am Thorac Soc 2018; 15: 281-289.

42 Rahaghi F, Come C, Ross J, et al. Morphologic response of the pulmonary vasculature to endoscopic lung volume reduction. Chronic Obstr Pulm Dis 2015; 2: 214-222. 